\title{
Infarto agudo de miocardio: revisión sobre factores de riesgo, etiología, hallazgos angiográficos y desenlaces en pacientes jóvenes
}

\author{
Acute myocardial infarction: Review on risk factors, etiologies, angiographic \\ characteristics and outcomes in young patients
}

\author{
Carlos A. Dattoli-García*, Cynthia N. Jackson-Pedroza, Andrea L. Gallardo-Grajeda, \\ Rodrigo Gopar-Nieto, Diego Araiza-Garygordobil y Alexandra Arias-Mendoza \\ Servicio de Urgencias y Unidad Coronaria, Instituto Nacional de Cardiología, Ciudad de México, México
}

\begin{abstract}
Resumen
La enfermedad cardiovascular persiste como primera causa mundial de muerte en los adultos. La población de adultos jóvenes ha cursado con cambios en el estilo de vida con el paso de las décadas, favoreciendo la aparición de ateroesclerosis en etapas más tempranas y como consecuencia la aparición de eventos cardiovasculares de manera más prematura. Se ha identificado que dentro de los factores de riesgo más comunes, la mayoría de ellos son potencialmente modificables. En comparación con adultos mayores, se ha identificado con mayor prevalencia la presencia de etiologías no ateroescleróticas de infarto de miocardio, como la disección coronaria espontánea, alteraciones anatómicas, embolia y espasmo coronarios. Los hallazgos angiográficos y desenlaces son diferentes de acuerdo con el grupo de edad y el sexo. Por dicho motivo realizamos una búsqueda en PubMed de los estudios y registros publicados para el estudio del infarto agudo de miocardio en paciente jóvenes. Con dicha información realizamos la presente revisión con el objetivo de una mejor comprensión de los hallazgos comunes en este grupo y realizar su comparación con grupos de mayor edad.
\end{abstract}

Palabras clave: Infarto agudo de miocardio. Jóvenes. Aterosclerosis. Enfermedad coronaria. Angiografía coronaria. Disección coronaria.

\begin{abstract}
Cardiovascular disease is the leading cause of death in adults around the world. Young adult population has suffered changes in lifestyle over the decades, favoring the appearance of atherosclerosis at early ages, and as a consequence, the incidence of cardiovascular events emerges prematurely. It has been identified that most common risk factors are potentially modifiable. There is a greater prevalence of non-atherosclerotic etiologies of myocardial infarction such as spontaneous coronary dissection, congenital malformations, coronary embolism, and coronary spasm. Different angiographic findings and outcomes according to age and gender have been recognized. For this reason, we searched PubMed for published research and registries for the study of acute myocardial infarction in young patients. With this data, we carried out the present review to better understand the common findings in this group, and to compare them with older age groups.
\end{abstract}

Key words: Myocardial infarct. Young patients. Atherosclerosis. Coronary artery disease. Coronary angiography.

\section{Correspondencia:}

${ }^{*}$ Carlos A. Dattoli-García

E-mail: carlos.dattolig@gmail.com
Fecha de recepción: 13-08-2020

Fecha de aceptación: 18-12-2020 DOI: 10.24875/ACM.20000386
Disponible en internet: 20-01-2021 Arch Cardiol Mex. 2021;91(4):485-492 www.archivoscardiologia.com

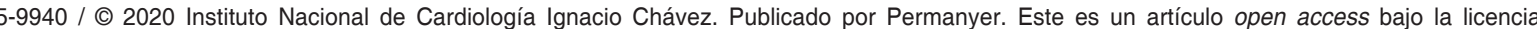
CC BY-NC-ND (http://creativecommons.org/licenses/by-nc-nd/4.0/). 


\section{Introducción}

En la actualidad es cada vez es más frecuente encontrar a personas jóvenes con diagnóstico de infarto agudo de miocardio (IAM), lo cual puede asociarse al incremento de factores de riesgo, tales como la sobrecarga de trabajo, estrés laboral, malos hábitos dietéticos, sedentarismo, obesidad, tabaquismo y adicciones. Es de llamar la atención que en algunos países se ha reportado disminución de la incidencia y mortalidad del IAM en las últimas décadas, sin embargo, esta tendencia no se ha observado en los jóvenes, ya que en este grupo etario se ha advertido un incremento en la incidencia ${ }^{1-4}$.

El término «infarto de miocardio en paciente joven» no se encuentra definido por guías o asociaciones, sin embargo, la mayoría de los estudios han establecido un punto corte menor de 40 a 45 años para identificar a este grupo de pacientes (Tabla 1). Diversos estudios han mostrado que del total de los pacientes con IAM, aproximadamente un 6 a $12 \%$ de los pacientes son menores de 45 años, el 3.4 a $5.6 \%$ son menores de 40 años y el $1.6 \%$ son menores de 35 años. A pesar de los avances en el tratamiento en los pacientes con IAM, el pronóstico en los pacientes jóvenes con IAM no se ha visto modificado significativamente, en especial la mortalidad en el sexo femenino 5,6,9-19.

La importancia de la identificación temprana de los factores de riesgo prevenibles reside en que es la piedra angular para disminuir eventos cardiovasculares prematuros, los cuales tienen un fuerte impacto en el estilo de vida y productividad, generando un gran impacto social y económico para la propia familia y la sociedad en general ${ }^{20}$.

La presente revisión tiene como objetivo establecer un marco conceptual de los factores de riesgo, la presentación clínica, etiología y desenlaces de IAM en personas jóvenes. La obtención de datos se efectuó en el mes de junio del 2020 por medio de la búsqueda en PubMed de los siguientes conceptos: myocardial infarction, young patients, age differences. Se seleccionaron los artículos en idioma español e inglés, publicados entre 1982 hasta el mes de mayo de 2020. Se seleccionaron aquellos que incluyeron a pacientes menores de 45 años de edad y que describieron factores de riesgo, hallazgos angiográficos o desenlaces cardiovasculares comparados con una población de mayor edad. Se realizó una síntesis de dichos hallazgos, la cual se encuentra descrita en el siguiente documento.

\section{Factores de riesgo}

Los factores de riesgo cardiovasculares fueron identificados a partir del estudio Framingham en EE.UU. en el año de $1948^{21}$. En la actualidad los factores de riesgo pueden clasificarse como modificables y no modificables; dicha clasificación permite identificar los cambios que se pueden hacer en el estilo de vida y el tratamiento del paciente para disminuir la aparición de eventos cardiovasculares y disminuir su mortalidad ${ }^{22}$. En un estudio retrospectivo en el que se incluyeron 3,501 pacientes de 18 a 55 años que fueron hospitalizados por IAM se observó que hasta el $97 \%$ de las mujeres y el $99 \%$ de los hombres tienen al menos un factor de riesgo potencialmente modificable, y el $65 \%$ de las mujeres y $63 \%$ de los hombres tenían tres o más ${ }^{23}$.

En diversos registros que incluyen a pacientes jóvenes ( $<45$ años) se ha identificado que los principales factores de riesgo en este grupo son: tabaquismo $(60-80 \%)$, dislipidemia (25-86\%), historia familiar de enfermedad coronaria prematura (20-56\%), obesidad (28$49 \%$ ), hipertensión (16-63\%), diabetes mellitus (4-35\%) y uso de drogas ilícitas $(5 \%)^{5,7,9,13,24-26}$. Al ser comparados con pacientes de 45-59 años y mayores de 60 años se encontró que en el grupo de pacientes jóvenes la presencia de tabaquismo (56 vs. 50 y $29 \%, p<0.001$ ), obesidad (30 vs. 17 y $27 \%, p<0.001$ ) y antecedente de muerte familiar prematura por causa cardiovascular (44 vs. 35 y 19\%, p< 0.001) fue mayor (Fig. 1) $)^{14,25}$.

En el análisis por sexo, se puede observar que la presencia de IAM prematuro es más frecuente en el masculino ${ }^{10-12,14,24,27}$; además en los hombres fue más frecuente encontrar antecedente de dislipidemia (5992.1 vs. $30-82.6 \%$ ) y tabaquismo (57 vs. $48 \%$ ), mientras que en el sexo femenino se observó más frecuentemente diabetes mellitus (15-39 vs. 10-26\%) ${ }^{14,17,18,23,28}$.

El registro YOUNG-MI mostró que aproximadamente un $9 \%$ de los pacientes con IAM menores de 50 años tenían probable o definitiva hipercolesterolemia familiar y solo el $42.8 \%$ estaba con tratamiento previo con estatina ${ }^{29}$.

En una cohorte retrospectiva que incluyó pacientes con IAM con elevación del segmento ST antes de los 50 años, se encontró que el $72 \%$ tenía una puntuación de riesgo cardiovascular ASCVD (atherosclerotic cardiovascular disease) menor del 7.5\%; sin embargo, cuando se consideró el riesgo cardiovascular vitalicio, el $80.3 \%$ estaba en el grupo de alto riesgo. Si se aplicaban las recomendaciones ACC/AHA (American College of Cardiology/American Heart Association) del 2013 y USPSTF (U.S. Preventive Services Task Force) 
Tabla 1. Registros reportados para el estudio de pacientes jóvenes con infarto agudo de miocardio

\begin{tabular}{|l|l|l|c|c|c|c|c|}
\hline Autor & Año & $\begin{array}{l}\text { Origen de la } \\
\text { población }\end{array}$ & $\begin{array}{c}\text { Total de } \\
\text { pacientes } \\
\text { con IAM }\end{array}$ & $\begin{array}{c}\text { Definición de } \\
\text { paciente joven }\end{array}$ & $\begin{array}{c}\text { Proporción de } \\
\text { pacientes } \\
\text { jóvenes }\end{array}$ & $\begin{array}{c}\text { Sexo } \\
\text { masculino }\end{array}$ & $\begin{array}{c}\text { Mortalidad } \\
\text { intrahospitalaria } \\
\text { en jóvenes }\end{array}$ \\
\hline Badui, et al., 19935 & 1993 & México & 2,525 & $<40$ años & $5.6 \%(142)$ & $87 \%$ & $3 \%$ \\
\hline Correa- Chacón, et al., 20036 & 2003 & México & 383 & $<40$ años & $3.4 \%(13)$ & $92 \%$ & No reportado \\
\hline Ruiz-Pizarro, et al., 20197 & 2018 & España & 3,883 & $<35$ años & $1.6 \%(61)$ & $88 \%$ & $8 \%$ \\
\hline Yang, et al., 2020 & 2019 & EE.UU. & 2,097 & $<40$ años & $20.5 \%(431)$ & $80 \%$ & $4.2 \%$ \\
\hline Vautrin, et al., 2020 & 2019 & Francia & - & $<45$ años & 489 & $88 \%$ & $2 \%$ \\
\hline Wittlinger, et al., 202010 & 2019 & Alemania & 5,873 & $<40$ años & $2 \%(119)$ & $79 \%$ & $2.5 \%$ \\
\hline Khoury, et al., 2020 & 2019 & Israel & 2,467 & $<35$ años & $1.86 \%(46)$ & $89 \%$ & $0 \%$ \\
\hline Batra, et al., 201912 & 2019 & Pakistán & 415 & $<40$ años & $12 \%(50)$ & $84 \%$ & $2 \%$ \\
\hline Zgheib, et al., 2020 & 2019 & Líbano & 2,884 & $<45$ años & $6.06 \%(175)$ & $93 \%$ & $1.87 \%$ \\
\hline Jortveit, et al., 202014 & 2020 & Noruega & 33,439 & $<45$ años & $4.4 \%(1,468)$ & $81 \%$ & $2 \%$ \\
\hline
\end{tabular}

IAM: infarto agudo de miocardio.

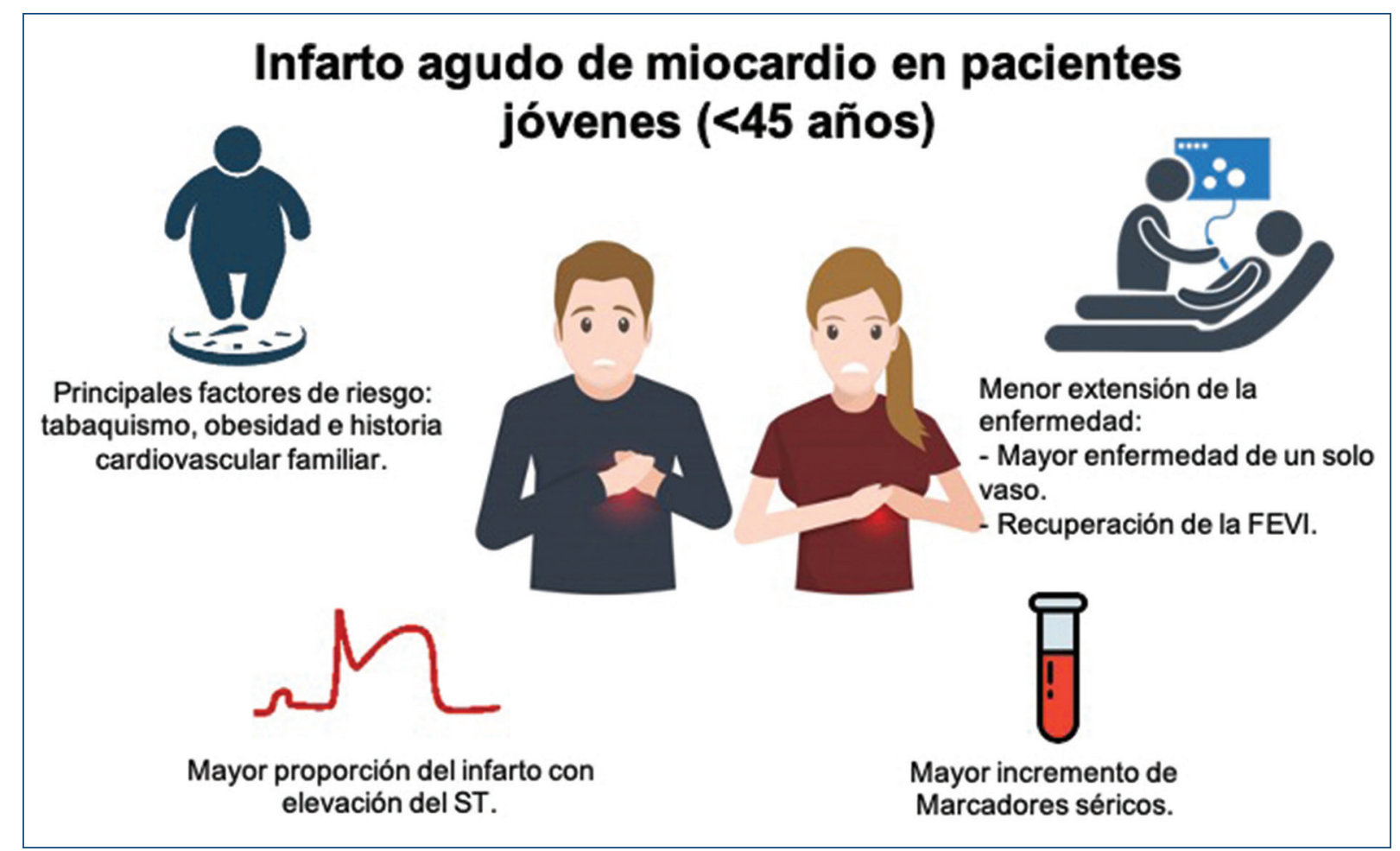

Figura 1. Principales características en los pacientes jóvenes con infarto de miocardio.

FEVI: fracción de eyección del ventrículo izquierdo.

del 2016 para indicación de estatinas, solo el 49 y el $29 \%$ eran categorizados como elegibles para tratamiento previo a su IAM. Con dichos resultados se puede concluir que dichas escalas no identifican adecuadamente al paciente joven para tratamiento con estatina previo a su evento, por lo que se deberán crear nuevas estrategias para su adecuada identificación ${ }^{28}$. 
En cuanto a factores genéticos, se ha demostrado que altas concentraciones del inhibidor de activación del plasminógeno tipo 1, la presencia de polimorfismos de nucleótidos simples, variantes en genes de paroxonasa 1, receptor toll-like tipo 4 y metaloproteinasa 3, están asociados a eventos trombóticos. Su estudio se ha realizado únicamente para fines de investigación y no se lleva a cabo de manera estándar en la población joven ${ }^{30-33}$.

\section{Presentación clínica}

El cuadro clínico es indistinguible del de los pacientes mayores. El tipo de presentación más frecuente es el dolor precordial típico en el $81-95 \%$, seguido de síntomas neurovegetativos en el $87 \%$ y paro cardiaco o arritmias letales en el $1.5-11 \% \%^{7,9,24,34}$. Una de las grandes diferencias es que en los pacientes jóvenes existe una menor proporción de antecedente de episodios de angina y que el tiempo de inicio de los síntomas al momento de la presentación hospitalaria tiende a ser menor ${ }^{10,34,35}$.

Una mayor proporción de pacientes jóvenes tiene un IAM con elevación del ST como presentación (70\%), mientras que los pacientes no jóvenes lo hacen tanto con IAM con elevación del ST como con IAM sin elevación del ST en la mitad de los casos. Por otra parte, se ha observado una mayor prevalencia de infarto de miocardio sin arterias coronarias obstruidas en los menores 45 años (14 vs. $9 \%, p<0.001$ ), con una mayor frecuencia en mujeres jóvenes ( 23 vs. $10 \%, p<0.001)^{10,14}$.

Es importante mencionar que en los jóvenes se observa un mayor pico de creatina cinasa y proteína $C$ reactiva durante el evento agudo, lo cual sugiere que el miocardio no tiene circulación colateral ${ }^{10,11}$; sin embargo, se debe reconocer que la elevación enzimática no siempre se corresponde con el curso clínico, debido a que al momento del ingreso de estos pacientes en su mayoría muestran escalas pronósticas con puntuación de mortalidad baja a moderada y de muy bajo riesgo de sangrado $0^{7,9,15}$.

\section{Etiología}

En la población general se ha observado que la ruptura de placa ateroesclerótica con consecuente trombosis es principal causa de IAM independientemente de su presentación clínica hasta en el $79 \%$ de los ca$\operatorname{sos}^{36,37}$. La presencia de ateroesclerosis en pacientes jóvenes no es un hallazgo poco común; se ha encontrado que en donadores de trasplante cardiaco con edad media de $33 \pm 13.2$ años y sin evidencia de enfermedad coronaria el $51.9 \%$ tenían presente al menos una placa ateroesclerótica documentada por ultrasonido coronario intravascular ${ }^{38}$. Otro estudio realizado en pacientes de $45.7 \pm 4.2$ años sin antecedente de enfermedad cardiovascular, se encontró que el $61.8 \%$ tenía ateroesclerosis evidenciada por métodos de imagen y en un seguimiento a tres años el $41.5 \%$ presentó progresión ${ }^{39}$. Estos hallazgos respaldan el hecho de que en los pacientes jóvenes con IAM la aterosclerosis persiste como la causa más frecuente, teniendo una

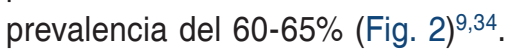

Otras etiologías como: disección, embolia, espasmo, alteraciones anatómicas y disfunción microvascular pueden ser encontradas en el $10-11 \%$ en los hombres y hasta en el $30 \%$ de las mujeres jóvenes. Este grupo de pacientes tiene un mejor pronóstico que aquellos con aterosclerosis como causa del IAM ${ }^{9,24,40}$.

La disección coronaria espontánea se observa en el $0.07-1.1 \%$ del total de los pacientes con IAM y en el $4.87 \%$ de los pacientes jóvenes; es más frecuente su presentación en mujeres jóvenes, ya que representa hasta un $22-35 \%$ del total de causas de IAM. Se asocia frecuentemente al embarazo, enfermedades del tejido conectivo e inflamatorias, y su principal causa es la displasia fibromuscular ${ }^{9,24,34,40,41}$.

La embolia coronaria puede representar la causa del $2.9 \%$ del total de los pacientes con IAM; su prevalencia en el grupo de pacientes jóvenes se desconoce. El material que es enviado a las arterias coronarias y produce su obstrucción puede ser de origen trombótico, tumoral, aire, vegetación o un cuerpo extraño $0^{40,42}$. Las principales causas son fibrilación auricular, cortocircuitos venoarteriales, miocardiopatía dilatada, enfermedad valvular, tumores cardiacos, estados hipercoagulables, endocarditis, tumores y causa iatrogénica.

En la actualidad no se ha establecido de manera estándar el abordaje de trombofilias en pacientes jóvenes con hallazgo de trombosis coronaria sin causa aterosclerótica, por lo que dicho abordaje debe ser acorde a la sospecha clínica. En algunos estudios se ha realizado tamizaje de trombofilia en IAM en menores de 35 años $\sin$ factores de riesgo convencionales, encontrando que un $3.2-4.7 \%$ fueron positivos para síndrome antifosfolípido y un $1.63 \%$ para deficiencia de proteína $C^{7,11}$.

El espasmo coronario es una entidad frecuente en poblaciones asiáticas y su prevalencia como causa de IAM en la población general y en el grupo de pacientes jóvenes se desconoce ${ }^{43}$. Se ha observado que en pacientes con IAM y enfermedad coronaria no obstructiva hasta un $33.4-44.8 \%$ presentan espasmo coronario inducido por prueba inductora ${ }^{44,45}$. 


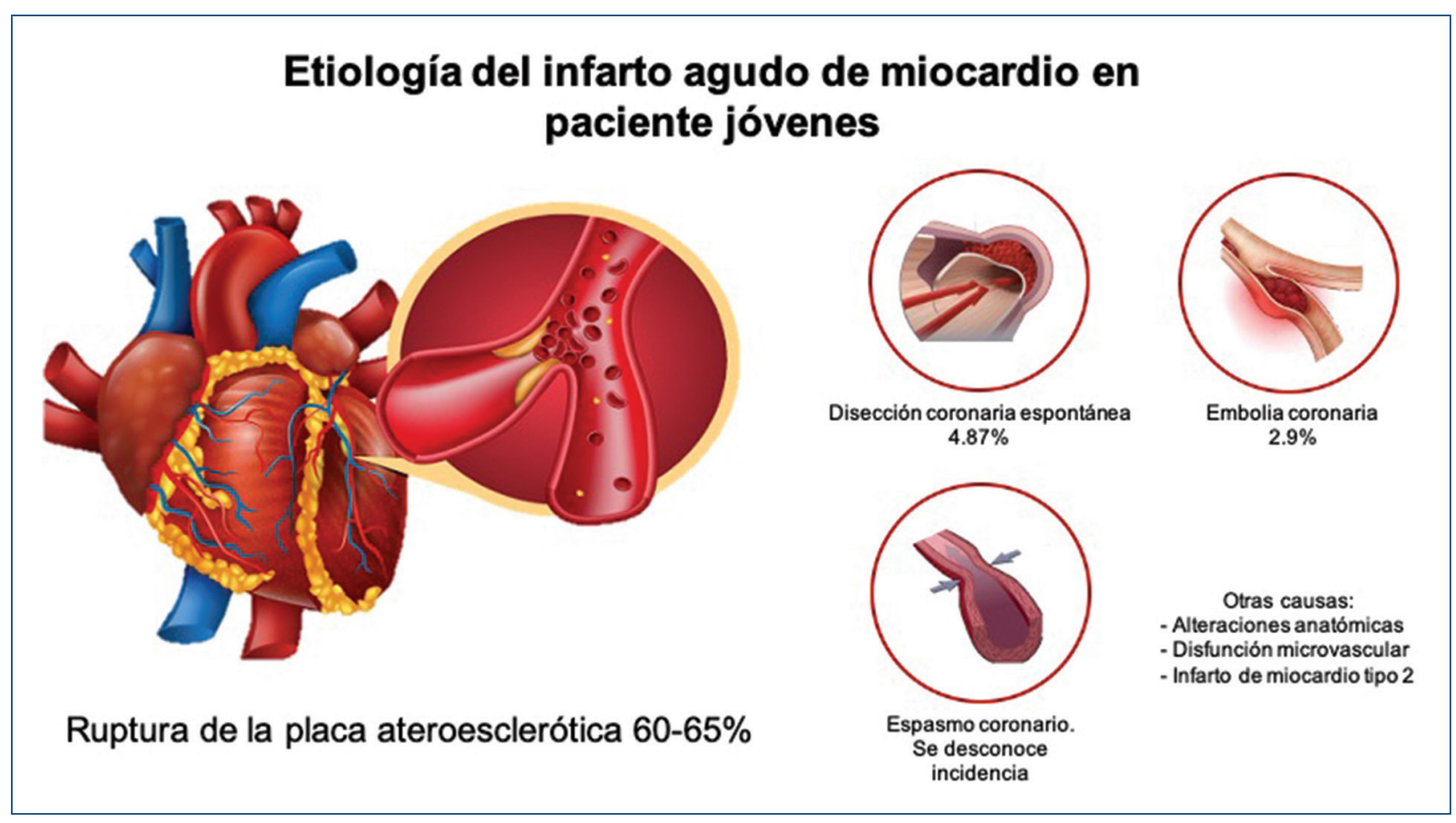

Figura 2. Esquema de las principales causas del infarto agudo de miocardio en pacientes jóvenes.

La prevalencia de las alteraciones anatómicas coronarias como causa de isquemia cardiaca no ha sido reportada, sin embargo, hay que destacar que las anomalías en el origen de las coronarias, así como su estenosis anatómica, pueden impedir el llenado diastólico, con la consecuente isquemia. Otra causa descrita es la presencia de un trayecto anómalo con disminución de su volumen por compresión extrínseca ${ }^{46,47}$.

Otra causa no ateroesclerótica de IAM es el desequilibrio entre el aporte y demanda de oxígeno al músculo cardiaco, comúnmente conocido con IAM tipo 2, el cual se presenta hasta en el $31.9 \%$ de los jóvenes con IAM. Las principales causas reportadas que provocan este desequilibrio son sepsis (16.2\%), arritmias (14.5\%) e insuficiencia respiratoria $(11.5 \%)^{15}$.

\section{Terapia de reperfusión y hallazgos angiográficos}

En la mayoría de los jóvenes se realiza coronariografía cuando se trata de IAM tipo 1 (94\%), sin embargo, se realiza con menor frecuencia en IAM tipo $2(22.6 \%)^{7}$. La terapia primaria de reperfusión elegida para los pacientes jóvenes con IAM con elevación del ST se realiza de acuerdo con la disponibilidad de intervención coronaria percutánea primaria o trombólisis en el centro de atención, sin embargo, durante la hospitalización hasta casi la totalidad es sometido a angiografía coronaria $7,9,10,24,48$.

Dentro de los hallazgos angiográficos en jóvenes, se ha reportado una mayor frecuencia la enfermedad de un vaso (53.7-82 vs. $31-50 \%)^{11-13,24,25,48}$. Por el contrario, la enfermedad de tres vasos es poco frecuente en el grupo de pacientes jóvenes (2.4-12\%) comparado con el grupo $>40$ años (hasta un $34.8 \%)^{12}$ (Fig. 3). El vaso más afectado es la arteria descendente anterior $(46-71.5 \%)^{7,9,10,12,13,24,48}$, seguida de la arteria coronaria derecha $(22-40 \%)^{7,12,13,24}$ y por último la arteria circunfleja $(7-9 \%)^{7,9,13}$.

El diagnóstico de IAM sin enfermedad coronaria obstructiva se encuentra en el 10.9-14\% de los jóvenes, siendo más frecuente en mujeres (23 vs. $10 \%)^{9,10}$.

No se ha observado diferencia en los tiempos puerta-dispositivo al comparar a los jóvenes con el grupo de mayor edad, sin embargo, algunos estudios mostraron un mayor uso de stents liberadores de fármaco en jóvenes (56.6 vs. $34.3 \%$ ) y más stents metálicos desnudos en los mayores (37.7 vs. $61.3 \%)^{10,14}$. Es de resaltar que no se ha observado diferencia en flujos TIMI (thrombolysis in myocardial infarction), carga de trombo, implante de stent y técnicas de intervención coronaria percutánea por sexo, ni en flujo TIMI postangioplastia entre edades ${ }^{9,12}$. Por último, la tasa de 


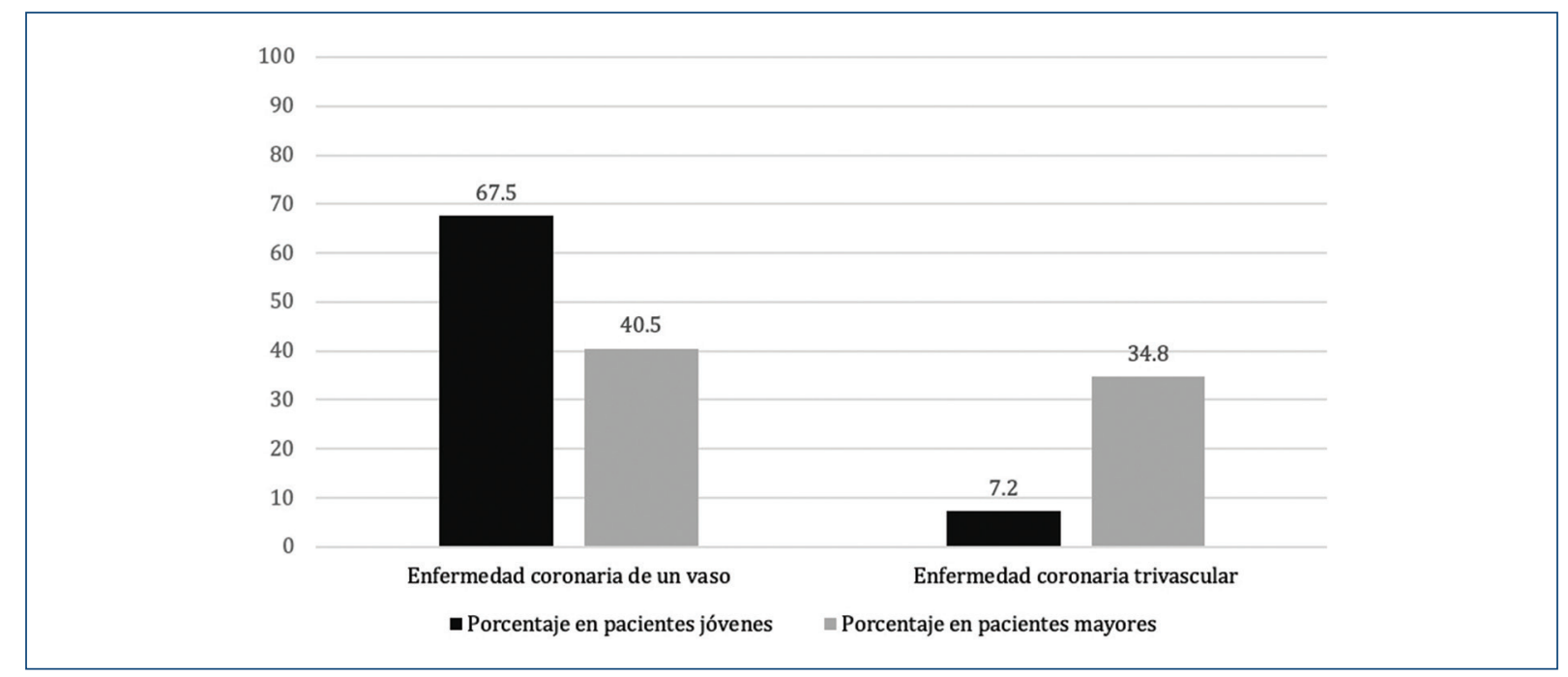

Figura 3. Comparación de hallazgos angiográficos en pacientes con infarto agudo de miocardio.

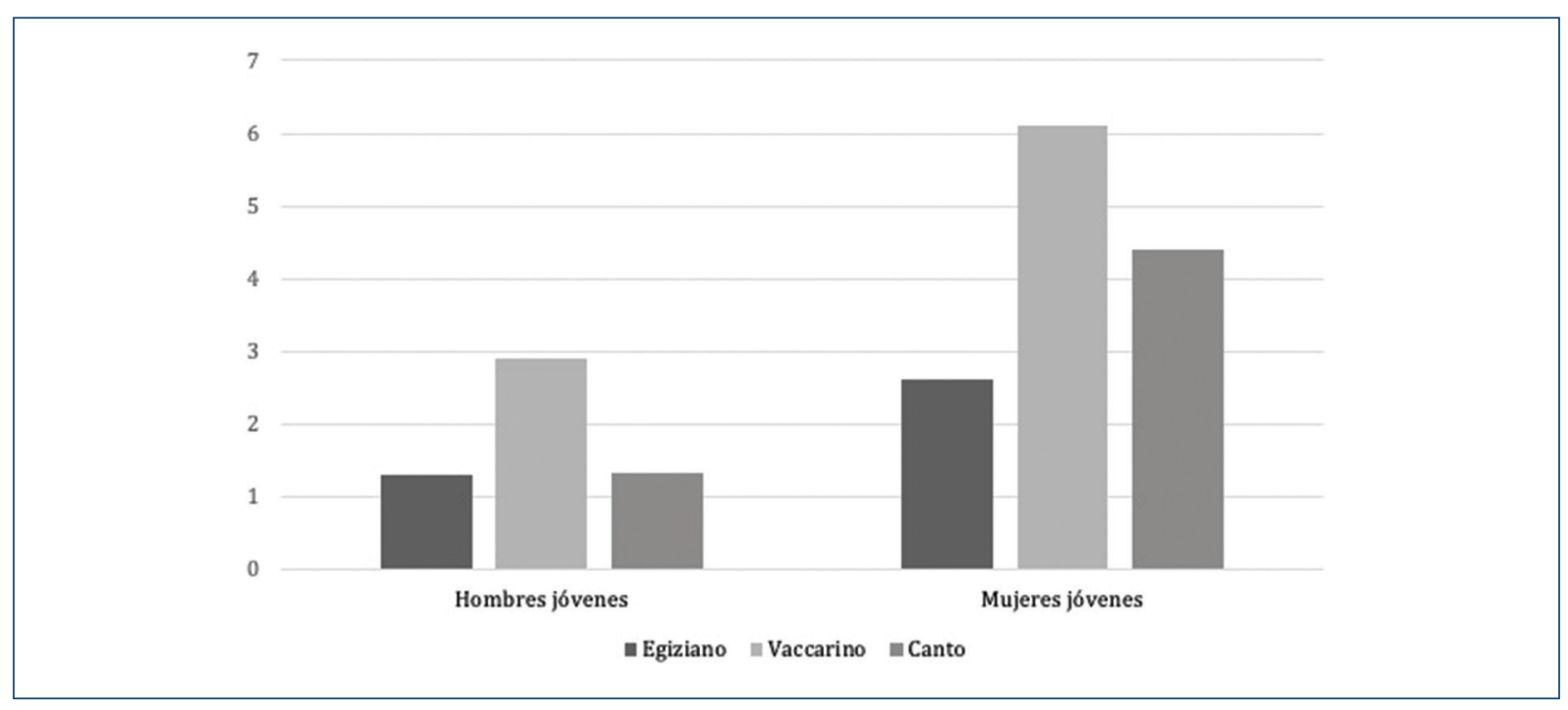

Figura 4. Mortalidad por sexo expresada en porcentaje en pacientes jóvenes con infarto agudo de miocardio.

cirugía de revascularización coronaria es del 1.7 al 2\% de los jóvenes comparada al $9.2 \%$ de los mayores $^{9,10}$.

\section{Pronóstico}

La mortalidad intrahospitalaria en jóvenes con IAM es del $0.93-4 \%$, sin observarse diferencia entre sexos ni en grupos de edad intermedia (45-65 años), a excepción de un registro que muestra mayor mortalidad

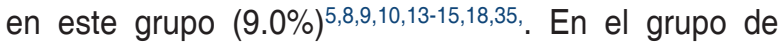
mayores de 65-70 años los estudios ha reportado una mortalidad intrahospitalaria mucho más elevada $(5-20 \%)^{14,35}$.

En cuanto a mortalidad a largo plazo, se encontró que este parámetro fue menor en $<45$ años comparado con 45-59 años y con 60-80 años ${ }^{14}$. El CASS (Estudio de Cirugía de la Arteria Coronaria) demostró mayor supervivencia a siete años en hombres < 35 años comparados con los $>35$ años ( 84 vs. $75 \%$ ), igualmente se observó menor mortalidad en mujeres $<45$ años comparando con las $>45$ años $(90 \text { vs. } 77 \%)^{25}$. Lamentablemente, al analizar por sexo en el grupo de los pacientes jóvenes se ha reportado que la mortalidad a 
un año es hasta dos veces mayor en mujeres, pudiendo estar asociada a la presentación de síntomas atípicos, con el consecuente retraso en el diagnóstico y tratamiento (Fig. 4) ${ }^{17,19,49}$.

Por otra parte, no se ha observado diferencia en la estancia hospitalaria en menores y mayores de 40 años. En cuanto a las complicaciones intrahospitalarias, se ha reportado una baja tasa de choque cardiogénico (3\%), insuficiencia cardiaca $(10 \%)$, evento vascular cerebral $(1.5 \%)$, bloqueo auriculoventricular completo $(1.5-3 \%)$, pericarditis postinfarto $(1.5-5 \%)$ y lesión renal $(1.5 \%)^{5,7,9,12,13}$.

Por último, es conocido que la fracción de eyección del ventrículo izquierdo (FEVI) es un factor pronóstico después de un $\operatorname{IAM}^{50}$ y se ha demostrado que los jóvenes tienen mayor fracción de eyección que los mayores de 40 años $^{10,12}$. Un estudio que incluyó 1,724 pacientes con IAM menores de 50 años demostró que solo el $29 \%$ de los pacientes tenían una $\mathrm{FEVI}<50 \%$ en el momento del evento; durante el seguimiento aproximadamente el $42 \%$ mostró una recuperación de la $\mathrm{FEVI}>50 \%$ asociada a una disminución de la mortalidad por todas las causas (hazard ratio [HR]: $0.12 ; p<0.001)$ y una disminución en la mortalidad cardiovascular (HR: 0.10; $p<0.025)^{51}$.

Actualmente es reconocido que en los pacientes en recuperación después de un IAM es frecuente la depresión, reportando síntomas depresivos en aproximadamente el $47 \%$ de los pacientes en los primeros tres meses post-IAM, con una edad promedio de 54 años ${ }^{52}$. La depresión se considera un factor de riesgo independiente para aumento de mortalidad, incluso después de un adecuado control de la extensión de enfermedad coronaria, tamaño de infarto y disfunción ventricular izquierda. La causa de depresión post-IAM se desconoce, se asocia a alteraciones en la función neuroendocrina, falta de adherencia terapéutica y efectos adversos cardiotóxicos de fármacos antidepresivos ${ }^{53}$.

\section{Conclusión}

En la actualidad el IAM es cada vez más frecuente en jóvenes y su principal factor de riesgo cardiovascular es el tabaquismo. Su presentación clínica no suele diferir de los pacientes de mayor edad y la aterosclerosis persiste como principal etiología, sin embargo, hay que tener en cuenta otras causas como disección coronaria espontánea, embolia, espasmo y alteraciones anatómicas. La angiografía coronaria en jóvenes tiende a revelar enfermedad coronaria menos extensiva en comparación con pacientes de mayor edad. La mortalidad intrahospitalaria y a largo plazo es menor que en pacientes de mayor edad. Es importante conocer las características de esta patología de acuerdo con el grupo etario, ya que su identificación es relevante para establecer una terapéutica adecuada y prevenir complicaciones a corto y largo plazo.

\section{Financiamiento}

Ninguno.

\section{Conflicto de intereses}

Los autores declaran no tener conflicto de intereses.

\section{Responsabilidades éticas}

Protección de personas y animales. Los autores declaran que para esta investigación no se han realizado experimentos en seres humanos ni en animales.

Confidencialidad de los datos. Los autores declaran que han seguido los protocolos de su centro de trabajo sobre la publicación de datos de pacientes.

Derecho a la privacidad y consentimiento informado. Los autores declaran que en este artículo no aparecen datos de pacientes

\section{Bibliografía}

1. World Heart Organization Statistics. [Fecha de consulta: 10 de Agosto 2020]. Disponible en: https://www.who.int/health-topics/cardiovascular-diseases/\#tab=tab_1

2. Sulo G, Igland J, Nygård $O$, Vollset $S E$, Ebbing M, Tell GS. Favourable trends in incidence of AMI in Norway during 2001-2009 do not include younger adults: a CVDNOR project. Eur J Prev Cardiol. 2014;21(11):1358-64.

3. Yeh RW, Sidney S, Chandra M, Sorel M, Selby JV, Go AS. Population trends in the incidence and outcomes of acute myocardial infarction. $\mathrm{N}$ Engl J Med. 2010;362(23):2155-65.

4. Sans S, Puigdefábregas A, Paluzie G, Monterde D, Balaguer-Vintró I. Increasing trends of acute myocardial infarction in Spain: the MONICA-Catalonia Study. Eur Heart J. 2005;26(5):505-15.

5. Badui E, Rangel A, Valdespino A, Graef A, Plaza A, Chávez E, et al. Infarto agudo del miocardio en adultos jóvenes. Presentación de 142 casos. Arch Inst Cardiol Mex. 1993;63(6):529-37.

6. Correa-Chacón A, Macías-Magadán M, Robledo-Ramírez R, Ramírez-García JF, Hernández-Lara JA. Infarto agudo al miocardio en pacientes jóvenes. Medicina interna de México. 2003;19(1).

7. Ruiz Pizarro V, Palacios-Rubio J, Cruz-Utrilla A, García-Arribas D, Pérez-Vizcayno MJ, Fernández-Ortiz A, et al. ST-elevation myocardial infarction in patients $\leq 35$ years of age. Am J Cardiol. 2019;123(6): 889-93.

8. Yang J, Biery DW, Singh A, Divakaran S, DeFilippis EM, Wu WY, et al. Risk factors and outcomes of very young adults who experience myocardial infarction: The Partners YOUNG-MI Registry. Am J Med. 2020;133(5):605-612.e1.

9. Vautrin $E$, Jean ABP, Fourny $M$, Marlière $S$, Vanzetto $G$, Bouvaist $H$, et al. Sex differences in coronary artery lesions and in-hospital outcomes for patients with ST-segment elevation myocardial infarction under the age of 45. Catheter Cardiovasc Interv. 2020;96(6):1222-30.

10. Wittlinger T, Seifert C, Simonis G, Gerlach M, Strasser RH. Prognosis in myocardial infarction of young patients: Results of a prospective registry. Int J Cardiol. 2020;300:1-6. 
11. Khoury S, Soleman M, Margolis G, Barashi R, Rozenbaum Z, Keren G, et al. Incidence, characteristics and outcomes in very young patients with ST segment elevation myocardial infarction. Coron Artery Dis. 2020;31(2):103-8.

12. Batra MK, Rizvi NH, Sial JA, Saghir T, Karim M. Angiographic characteristics and in hospital outcome of young patients, age up to 40 versus more than 40 years undergoing primary percutaneous coronary intervention. J Pak Med Assoc. 2019:69(9):1308-12.

13. Zgheib H, Al Souky N, El Majzoub I, Wakil C, Sweidan K, Kaddoura R, et al. Comparison of outcomes in ST-elevation myocardial infarction according to age. Am J Emerg Med. 2020;38(3):485-90.

14. Jortveit J, Pripp AH, Langørgen J, Halvorsen S. Incidence, risk factors and outcome of young patients with myocardial infarction. Heart. 2020;106(18):1420-6.

15. Singh A, Gupta A, DeFilippis EM, Qamar A, Biery DW, Almarzooq Z, et al. Cardiovascular mortality after type 1 and type 2 myocardial infarction in young adults. J Am Coll Cardiol. 2020;75(9):1003-13.

16. Krumholz HM, Wang Y, Chen J, Drye EE, Spertus JA, Ross JS, et al. Reduction in acute myocardial infarction mortality in the United States: risk-standardized mortality rates from 1995-2006. JAMA. 2009;302(7): 767-73.

17. Egiziano G, Akhtari S, Pilote L, Daskalopoulou SS; GENESIS (GENdEr and Sex DetermInants of Cardiovascular Disease) Investigators. Sex differences in young patients with acute myocardial infarction. Diabe Med. 2013;30(3):e108-14.

18. Arora S, Stouffer GA, Kucharska-Newton AM, Qamar A, Vaduganathan M, Pandey A, et al. Twenty year trends and sex differences in young adults hospitalized with acute myocardial infarction. Circulation. 2019:139(8):1047-56

19. Vaccarino V, Parsons L, Every NR, Barron HV, Krumholz HM. Sex-based differences in early mortality after myocardial infarction. National Registry of Myocardial Infarction 2 Participants. N Engl J Med. 1999;341(4): 217-25.

20. Jain M, Sonathi V, Rathi H, Thomas SK, Mollon P. The economic impact of cardiovascular events in patients post myocardial infarction: Uk health care perspective. Value Health. 2014;17(7):A483-4.

21. Dawber TR, Meadors GF, Moore FEJ. Epidemiological approaches to heart disease: the Framingham Study. Am J Public Health Nations Health. $1951: 41(3): 279-81$

22. Yusuf $S$, Joseph $P$, Rangarajan $S$, Islam $S$, Mente A, Hystad $P$, et al Modifiable risk factors, cardiovascular disease, and mortality in 155722 individuals from 21 high-income, middle-income, and low-income countries (PURE): a prospective cohort study. Lancet. 2020;395(10226): 795-808

23. Leifheit-Limson EC, D'Onofrio G, Daneshvar M, Geda M, Bueno H, Spertus JA, et al. Sex differences in cardiac risk factors, perceived risk, and health care provider discussion of risk and risk modification among young patients with acute myocardial infarction: The VIRGO study. J Am Coll Cardiol. 2015;66(18):1949-57.

24. Deshmukh PP, Singh MM, Deshpande MA, Rajput AS. Clinical and angiographic profile of very young adults presenting with first acute myocardial infarction: Data from a tertiary care center in Central India. Indian Heart J. 2019:71(5):418-21.

25. Zimmerman FH, Cameron A, Fisher LD, Ng G. Myocardial infarction in young adults: angiographic characterization, risk factors and prognosis (Coronary Artery Surgery Study Registry). J Am Coll Cardiol. 1995;26(3):654-61.

26. Patel RS, Manocha P, Patel J, Patel R, Tankersley WE. Cannabis use is an independent predictor for acute myocardial infarction related hospitalization in younger population. J Adolesc Health. 2020;66(1):79-85

27. Schatz U, Fischer S, Müller G, Tselmin S, Birkenfeld AL, Julius U, et al Cardiovascular risk factors in patients with premature cardiovascular events attending the University of Dresden Lipid Clinic. Atheroscler Suppl. 2019;40:94-9.

28. Singh A, Collins BL, Gupta A, Fatima A, Qamar A, Biery D, et al. Cardiovascular risk and statin eligibility of young adults after an Ml: Partners YOUNG-MI Registry. J Am Coll Cardiol. 2018;71(3):292-302.

29. Singh A, Gupta A, Collins BL, Qamar A, Monda KL, Biery D, et al. Familial hypercholesterolemia among young adults with myocardial infarction. J Am Coll Cardiol. 2019;73(19):2439-50.
30. Nordt TK, Lohrmann J, Bode C. Regulation of PAl-1 expression by genetic polymorphisms. Impact on atherogenesis. Thromb Res. 2001;103(Suppl 1):S1-5.

31. Yamac AH, Uysal O, Ismailoglu Z, Ertürk M, Celikten M, Bacaksiz A et al. Premature myocardial infarction: Genetic variations in SIRT1 affect disease susceptibility. Cardiol Res Pract. 2019;2019:8921806

32. Gibbons GH, Liew CC, Goodarzi MO, Rotter JI, Hsueh WA, Siragy HM, et al. Genetic markers: progress and potential for cardiovascular disease. Circulation. 2004;109(25 Suppl 1):IV47-58.

33. Lusis AJ, Mar R, Pajukanta P. Genetics of atherosclerosis. Annu Rev Genomics Hum Genet. 2004:5:189-218.

34. Gulati R, Behfar A, Narula J, Kanwar A, Lerman A, Cooper L, et al. Acute myocardial infarction in young individuals. Mayo Clin Proc. 2020;95(1):136-56.

35. Hoit BD, Gilpin EA, Henning $H$, Maisel AA, Dittrich $H$, Carlisle J, et al. Myocardial infarction in young patients: an analysis by age subsets. Circulation. 1986;74(4):712-21.

36. Bentzon JF, Otsuka F, Virmani R, Falk E. Mechanisms of plaque formation and rupture. Circ Res. 2014;114(12):1852-66.

37. Libby P, Buring JE, Badimon L, Hansson GK, Deanfield J, Bittencourt MS, et al. Atherosclerosis. Nat Rev Dis Primers. 2019;5(1):56.

38. Tuzcu EM, Kapadia SR, Tutar E, Ziada KM, Hobbs RE, McCarthy PM, et al. High prevalence of coronary atherosclerosis in asymptomatic teenagers and young adults: evidence from intravascular ultrasound. Circulation. 2001;103(22):2705-10.

39. López-Melgar B, Fernández-Friera L, Oliva B, García-Ruiz JM, Sánchez-Cabo F, Bueno $\mathrm{H}$, et al. Short-term progression of multiterritorial subclinical atherosclerosis. J Am Coll Cardiol. 2020;75(14):1617-27.

40. Waterbury TM, Tarantini G, Vogel B, Mehran R, Gersh BJ, Gulati R. Non-atherosclerotic causes of acute coronary syndromes. Nat Rev Cardiol. 2020;17(4):229-41.

41. Tweet MS, Hayes SN, Pitta SR, Simari RD, Lerman A, Lennon RJ, et al. Clinical features, management, and prognosis of spontaneous coronary artery dissection. Circulation. 2012;126(5):579-88.

42. Shibata T, Kawakami S, Noguchi T, Tanaka T, Asaumi $Y$, Kanaya T, et al. Prevalence, clinical features, and prognosis of acute myocardial infarction attributable to coronary artery embolism. Circulation. 2015;132(4):241-50.

43. Beltrame JF, Sasayama S, Maseri A. Racial heterogeneity in coronary artery vasomotor reactivity: differences between Japanese and Caucasian patients. J Am Coll Cardiol. 1999;33(6):1442-52.

44. Bertrand ME, LaBlanche JM, Tilmant PY, Thieuleux FA, Delforge MR, Carre AG, et al. Frequency of provoked coronary arterial spasm in 1089 consecutive patients undergoing coronary arteriography. Circulation. 1982;65(7):1299-306.

45. Ong $\mathrm{P}$, Athanasiadis A, Hill S, Vogelsberg H, Voehringer M, Sechtem U. Coronary artery spasm as a frequent cause of acute coronary syndrome: The CASPAR (Coronary Artery Spasm in Patients With Acute Coronary Syndrome) Study. J Am Coll Cardiol. 2008;52(7):523-7.

46. Basso C, Maron BJ, Corrado D, Thiene G. Clinical profile of congenital coronary artery anomalies with origin from the wrong aortic sinus leading to sudden death in young competitive athletes. J Am Coll Cardiol. 2000;35(6):1493-501.

47. Van der Schoot GGF, Anthonio RL, Jessurun GAJ. Acute myocardial infarction in adolescents: reappraisal of underlying mechanisms. Neth Heart J. 2020;28(6):301-8.

48. Sozzi FB, Danzi GB, Foco L, Ferlini M, Tubaro M, Galli M, et al. Myocardial infarction in the young: a sex-based comparison. Coron Artery Dis. 2007;18(6):429-31

49. Canto JG, Rogers WJ, Goldberg RJ, Peterson ED, Wenger NK, Vaccarino $\mathrm{V}$, et al. Association of age and sex with myocardial infarction symptom presentation and in-hospital mortality. JAMA. 2012;307(8):813-22.

50. Candell Riera J. Estratificación pronóstica tras infarto agudo de miocardio. Rev Esp Cardiol. 2003:56(3):303-13.

51. Wu WY, Biery DW, Singh A, Divakaran S, Berman AN, Ayuba G, et al. Recovery of left ventricular systolic function and clinical outcomes in young adults with myocardial infarction. J Am Coll Cardiol. 2020;75(22):2804-15.

52. Denollet J, Sys SU, Brutsaert DL. Personality and mortality after myocardial infarction. Psychosom Med. 1995;57(6):582-91.

53. Ziegelstein RC. Depression after myocardial infarction. Cardiol Rev. $2001 ; 9(1): 45-51$. 